\title{
Optimization of ship's cathodic protection system to minimize electric and magnetic signature by mathematical simulation
}

\author{
S. Xing ${ }^{1,2}$, J. Wu ${ }^{1,2} \&$ Y. Yan ${ }^{1,2}$ \\ ${ }^{I}$ State Key Laboratory for Marine Corrosion and Protection, PR China \\ ${ }^{2}$ Luoyang Ship Material Research Institute, PR China
}

\begin{abstract}
In this contribution, the protective effect and distribution of magnetic field of cathodic protection $(\mathrm{CP})$ systems designed by experience were calculated by boundary element method (BEM) and the calculation results agreed with the measured results when the ship berthed at port, which suggested that the calculation results are accurate. The performance of a CP system was optimized by moving the position of the anodes as well as changing the output current of anodes. The objective was to design the system so that the electric and magnetic field was minimized and adequate protection was provided. The calculation results show that when the anodes were symmetrically installed at the $130^{\text {th }}$ and $232^{\text {nd }}$ frame, the corrosion related magnetic field and total output current were reduced by about $40 \%$ and $8.6 \%$ respectively.
\end{abstract}

Keywords: cathodic protection, mathematical simulation, boundary element method, electric and magnetic field.

\section{Introduction}

Coatings combined with impressed current cathodic protection (ICCP) is the most common means for the protection of shipboard. They interact with each other to protect shipboard. Coatings provide primary corrosion protection by isolating the hull metal from the seawater, while ICCP provides secondary corrosion protection in those areas where the paint is damaged or degrade. The protective effect is directly related to the ICCP configurations; an incorrectly designed ICCP system 
would not only adversely influence the protective effect but also the distribution of underwater electrical potential (UEP) and corrosion related magnetic (CRM) field, which are known as the signatures of the ship.

Electric fields and coincident magnetic fields, arise around a ship due to the current flow from the ICCP system. UEP and CRM signatures exist even in the absence of a cathodic protection system. They are caused by the galvanic potential differences between the metallic structures in contact with the seawater. For example, hull and propeller provides a sufficient driving potential to create an electric field. These electromagnetic fields take the form of steady electric and steady magnetic fields arising from the steady flow of current around the hull of the vessel. Modern mines can detect these fields and use them to detect and classify passing ships.

Research [1-4] suggests that design and optimization of the ICCP system is an effective means of preserving the integrity of a vessel and of controlling the signatures. The design of an optimized ICCP system requires accurate placement of the anodes and reference electrodes on the ship hull. Many shipboard ICCP systems currently in use were designed using relationships and engineering judgment. However, the protection effect of the $\mathrm{CP}$ system designed by experience cannot be known and sometimes it might lead to under or over protection and high electric signature. Today computer simulation techniques based on BEM like BEASY have enabled the electric and magnetic fields generated by the galvanic interaction of the ship metallic structure and ICCP system to be predicted. Thus, BEASY provides a tool to predict changes in the protection level of the ship and the electric field in the seawater caused by the ICCP system

In this paper, BEASY was used to predict and optimize the distribution of potential and electromagnetic field signature.

\section{Experimental work}

\subsection{Model}

A vessel with a length of 125 meters was investigated. The ship had 250 frames at intervals of $0.5 \mathrm{~m}$ and the frames were assigned the numbers 0 to 250 from bow to stern. The geometry of interest in the boundary element model was the wetted surface of the hull and major appendages. The ship has two propellers and two rudders; the propellers were made of nickel-aluminium-bronze alloy (NAB) and modelled as solid disks with equivalent surface area as the real propellers. The shaft is made of carbon steel and the propellers and shafts were assumed to be uncoated because of turbulence engendered by propeller movement. The ship hull and rudders are also made of carbon steel, which were coated to prevent corrosion. The ICCP system evaluated included four anodes and a centre controlled power supply. The BEM model of half of the ship is shown in Fig. 1. 


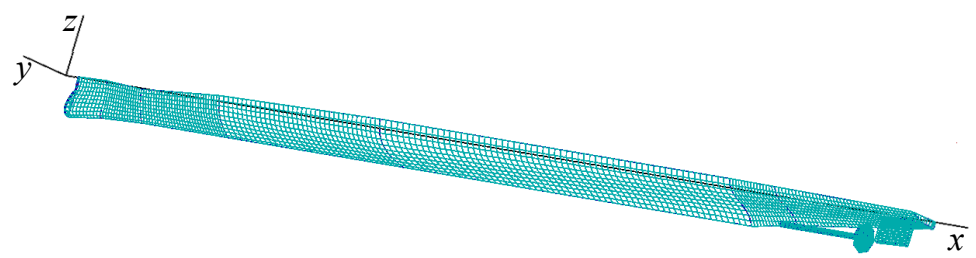

Figure 1: BEM model of the ship.

\subsection{Boundary conditions}

The cathodic polarization curves of uncoated and coated carbon steel are shown in Fig. 2(a) and the polarization curve of NAB is shown in Fig. 2(b); these curves were set as the boundary conditions of the ship model. During calculation, the polarization curve of coated steel was set as the boundary condition of ship hull and rudder, the polarization curve of uncoated steel was set as the boundary condition of shafts and the polarization curve of NAB was set as the boundary condition of propellers. A box of 2500 meters was created as the infinite boundary where the current density was zero.

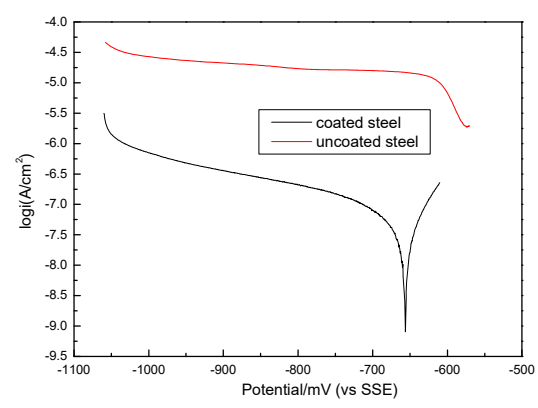

(a) Carbon steel

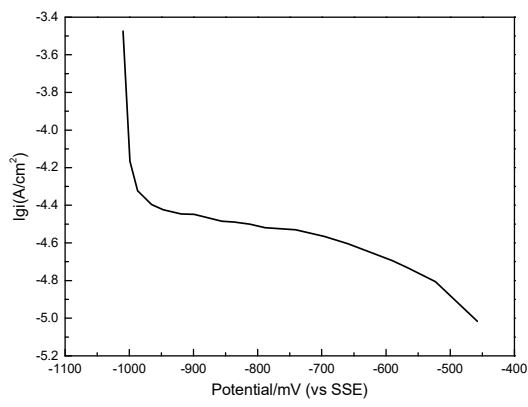

(b) NAB

Figure 2: Polarisation curves used as boundary conditions.

\subsection{ICCP optimization}

The optimization process required the problem to be posed in the form of an objective function, design variables and constraints. The sum of the squares of the differences between the potential on the element of the surfaces and the potential target value of the surfaces was used as the objective function to be minimized $[5,6]$ :

$$
O b j=\sum_{i=1}^{n_{s}} \sum_{j=1}^{n_{e}}\left(\phi_{i j}-\phi_{\text {target }, i}\right)^{2}
$$

subject to the following constraints on the surface of the cathode $\Gamma_{C}$ [3]: 


$$
\begin{gathered}
g_{i}=\frac{\phi_{i}-\phi_{\max , i}}{\left|\phi_{\min , i}\right|} \leq 0 \quad \text { on } \Gamma_{C}, \quad i=1,2, \ldots, n \\
g_{j}=\frac{\phi_{\min , j}-\phi_{j}}{\left|\phi_{\min , j}\right|} \leq 0 \quad \text { on } \Gamma_{C}, \quad j=1,2, \ldots, n
\end{gathered}
$$

where $n_{s}$ is the number of surfaces, $n_{e}$ is the number of elements per surface, $\phi_{\text {target }}$ is the target potential per surface, $\phi_{\max , j}$ and $\phi_{\min , j}$ are the maximum and minimum protection potentials, respectively.

\subsection{Magnetic fields}

The CRM signature generated by the currents flowing in the sea water could be calculated by solving the equation:

$$
\nabla^{2} \mathbf{A}=-\mu \mathbf{J}
$$

for the vector potential $\mathbf{A}$ [1] where $\mathbf{J}$ is the current density vector and $\mu$ the permeability of the medium. The magnetic field is then simply $\mathbf{B}=\operatorname{curl} \mathbf{A}$.

\section{Results and discussion}

\subsection{Performance of initial ICCP system on shipboard}

On the vessel, a single zone, four anodes ICCP system was used to protect the hull and appendages. The anodes were symmetrically installed at the $142^{\text {nd }}$ frame and $242^{\text {nd }}$ frame and the initial input current was $8 \mathrm{~A}$ and $10 \mathrm{~A}$, respectively. A saturated sulphate reference electrode (SSE) was installed at the $186^{\text {th }}$ frame. With the reference electrode reading of $-850 \mathrm{mV}$ (vs SSE), the potential distribution on the hull is shown in Fig. 3. The calculation results indicate that the hull, propellers and rudders were under protected, the potential range in the hull was from $-846 \mathrm{mV}$ to $-1000 \mathrm{mV}$. The current output of anode at the $142^{\text {nd }}$ frame and the $242^{\text {nd }}$ frame was 5.74 A and 7.40 A, respectively.

At the same time, the practical ship protective potential of the $37^{\text {th }}, 72^{\text {nd }}, 128^{\text {th }}$, $168^{\text {th }}$ and $228^{\text {th }}$ frame was measured when the ship berthed at port by $\mathrm{Cu} / \mathrm{CuSO}_{4}$ reference electrode. Compared with the measured results, the calculation results showed the same trend as the practical measured data and were near the same values as the measured results, as shown in Fig. 4. Thus, the calculation results appear to be accurate.

The CRM field distribution at sea level is shown in Fig. 5, where the $x$ axis is parallel with the keel of the ship and the $y$ axis is vertical to the keel. The keel of the ship was at the position $y=0 \mathrm{~m}$. The calculation results indicated that the magnetic field position with most strength was the region around the $142^{\text {nd }}$ frame where anodes were installed and then was the position of stern around the $240^{\text {th }}$ frame, while the normal of the magnetic field at the two positions was inverted. In Figs. 5, 7 and 9, the same colour denotes the same strength of the magnetic field and constant colour represents the same gradient of magnetic field. 


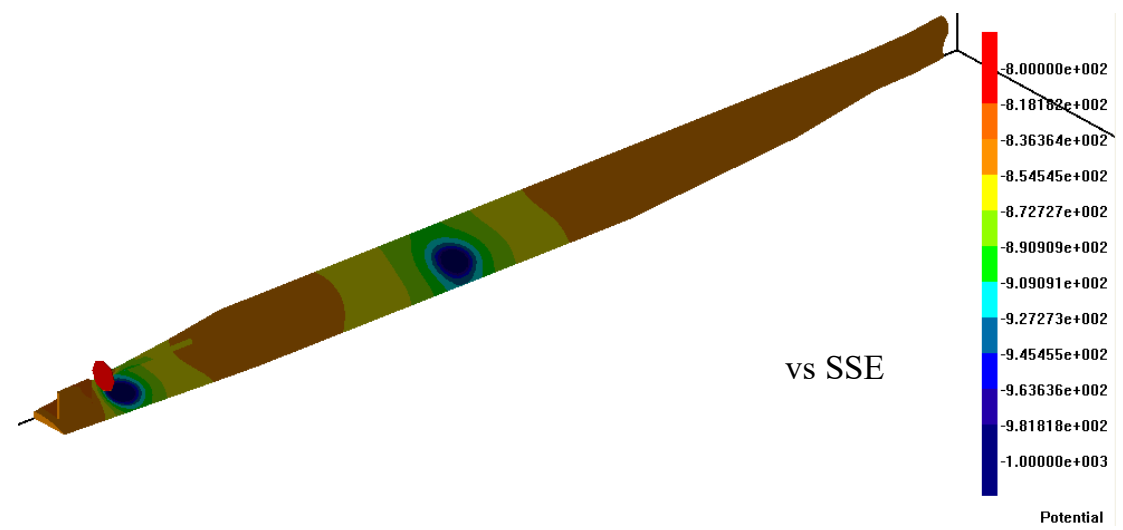

Figure 3: Potential plot of vessel protected by initial ICCP system.

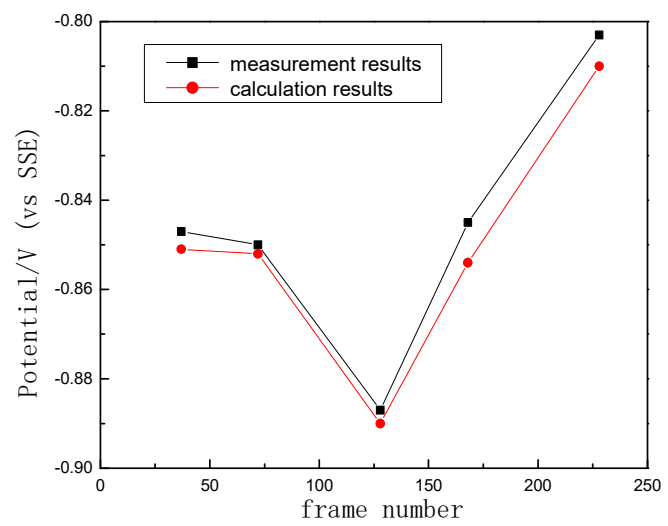

Figure 4: Potential vs frame number.

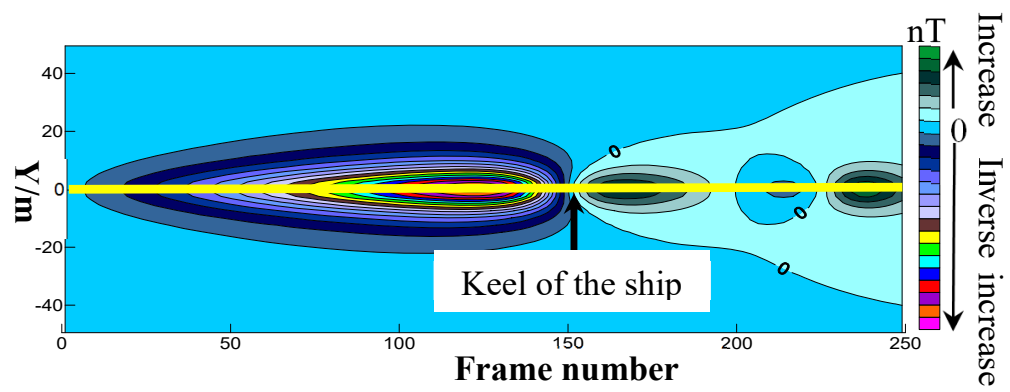

Figure 5: CRM field generated by initial ICCP system. 


\subsection{ICCP system optimization}

\subsubsection{Anodes position optimization}

Based on an empirically designed ICCP system, one group of anodes was set at the $232^{\text {th }}$ frame because the propellers consume more current. The other group of anodes was initially set at the $115^{\text {th }}$ frame, the candidate positions were set at the $115^{\text {th }}, 120^{\text {th }}, 130^{\text {th }}, 135^{\text {th }}, 142^{\text {nd }}$ and $150^{\text {th }}$ frame. By comparing the potential and magnetic field distribution of the ICCP system with different anodes setting, the best ICCP system was identified, in which the anodes were installed at the $130^{\text {th }}$ frame and $232^{\text {nd }}$ frame. When the initial input current of frame 1 and frame 2 was 5.7 $\mathrm{A}$ and 7.4 A, respectively, and with a reference electrode reading of $-850 \mathrm{mV}$ vs SSE, the potential distribution of ship hull ranged from $-821 \mathrm{mV}$ to $-1000 \mathrm{mV}$ as shown in Fig. 6. The current output of anode at the $130^{\text {nd }}$ and $232^{\text {nd }}$ frame was 5.32 A and 7.51 A, respectively.

After optimization, the CRM field distribution at sea level is shown in Fig. 7. In that figure, it can be seen that the position of maximum magnetic field strength changed to the $130^{\text {th }}$ frame, the position of relative strong magnetic field changed to the $160^{\text {th }}$ frame. Compared with the CRM distribution of initial ICCP system, by moving the position of anode, although the CRM field strength increased at stern, the magnetic field along the ship was improved as indicated in Fig. 8, which shows the magnetic field distribution at $y=0$ from bow to stern. The magnetic signature increase between $150^{\text {th }}$ frame and $200^{\text {th }}$ frame may be related to the distance increase between the two groups of anodes. The distance increase resulted in the current density increase between them, which led to the magnetic signature increasing. Through anode position optimization, the magnetic field strength was reduced by about $30 \%$.

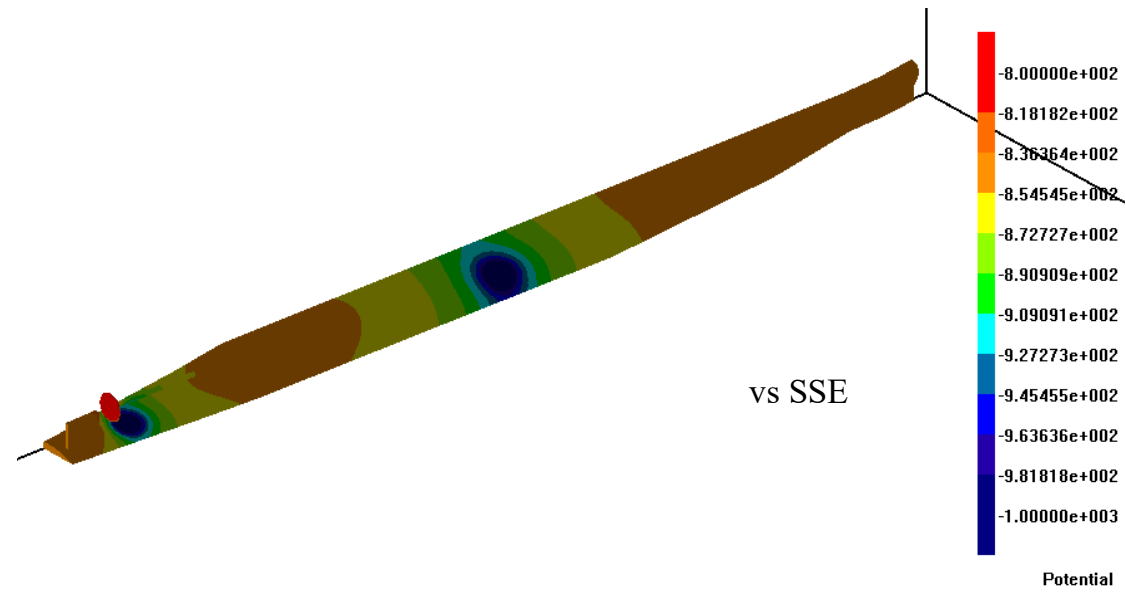

Figure 6: Potential plot of vessel protected by optimized anodes position ICCP system. 


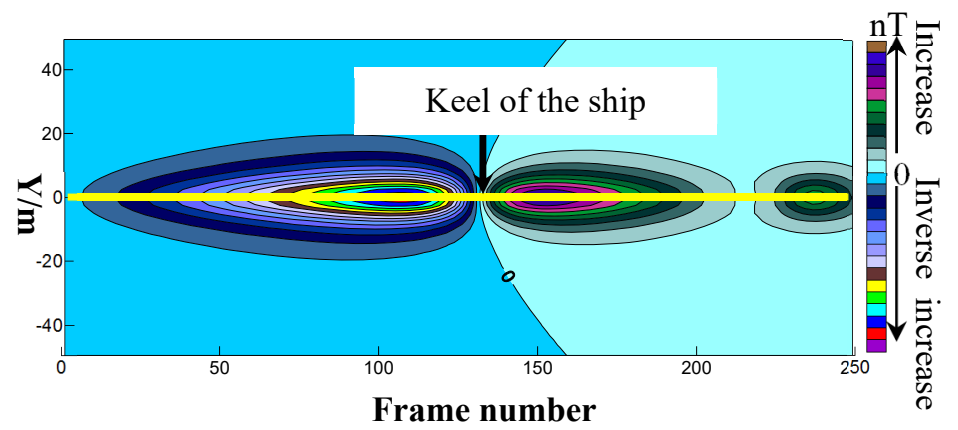

Figure 7: CRM field distribution after anodes position optimization.

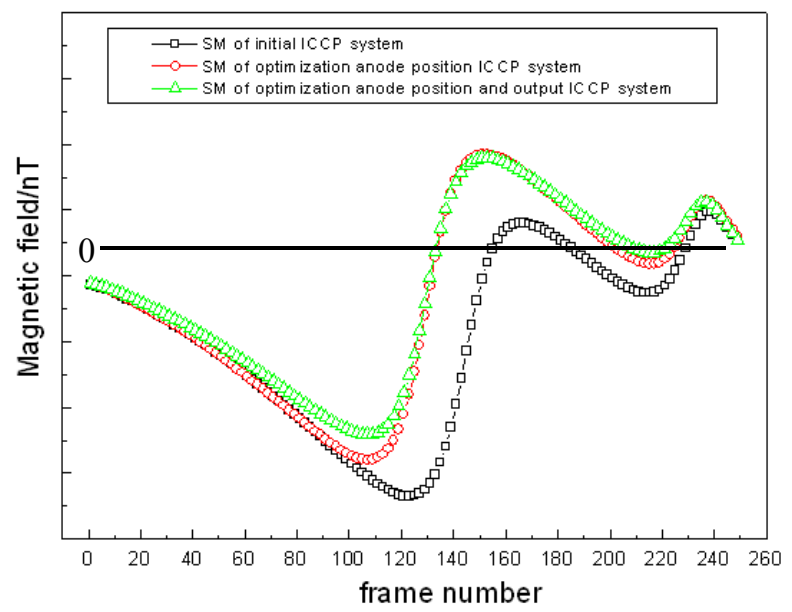

Figure 8: CRM field distributions at $y=0 \mathrm{~m}$.

\subsubsection{Anodes output current optimization}

Once the position of anodes was fixed, the optimization of anode output current was carried out. The purpose of optimizing the current was to minimize the corrosion related electromagnetic signature while the hull was provided with a sufficient cathodic protection effect. The initial input current of frame1 and frame2 was 5.3 A and 7.5 A, respectively. After current optimization, the obtained potential distribution on the hull is shown in Fig. 9. Compared with Figs. 3 and 5, the protective potential obviously changed in a more positive direction after current optimization, but the protective potential remained lower than $-800 \mathrm{mV}$, while the current output of anodes at the $130^{\text {th }}$ and $232^{\text {nd }}$ frame was minimized to 5.08 A and 6.93 A, respectively. Thus, through anode position and output current optimization, the output current was reduced by $8.6 \%$. 


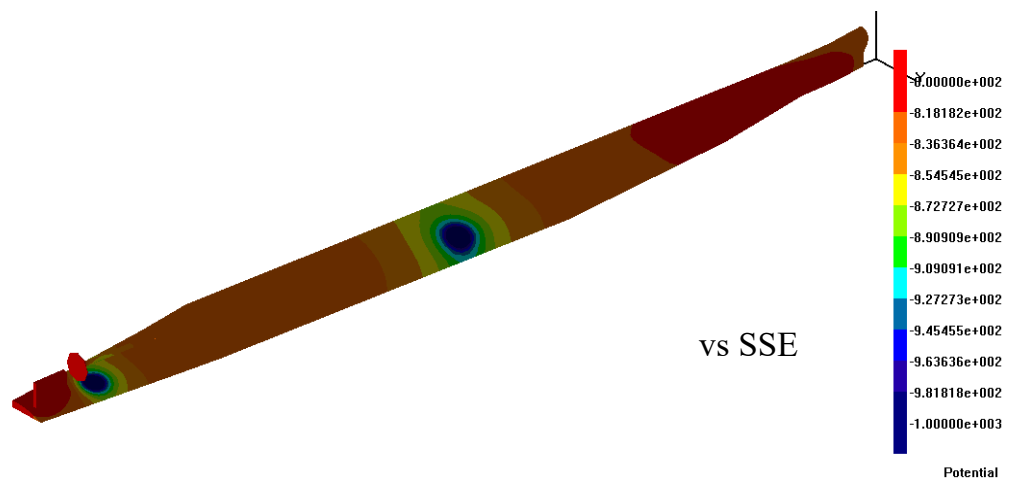

Figure 9: Potential distribution after anodes position and output current optimization.

The magnetic field was further reduced after current optimization as shown in Fig. 10. Compared with the magnetic field generated by the initial ICCP system, the strength of magnetic field was reduced by about $40 \%$. Like the magnetic field of the ICCP system optimized with respect to the anode position, the magnetic field around the position where the anode was installed was the strongest. In order to exhibit more intuitively the magnetic field distribution of initial and optimized ICCP systems, the magnetic signature at $y=0$ is shown in Fig. 8. In this figure, it can distinctly be seen that the magnetic field along the ship was step by step reduced by position and current of anodes optimization.

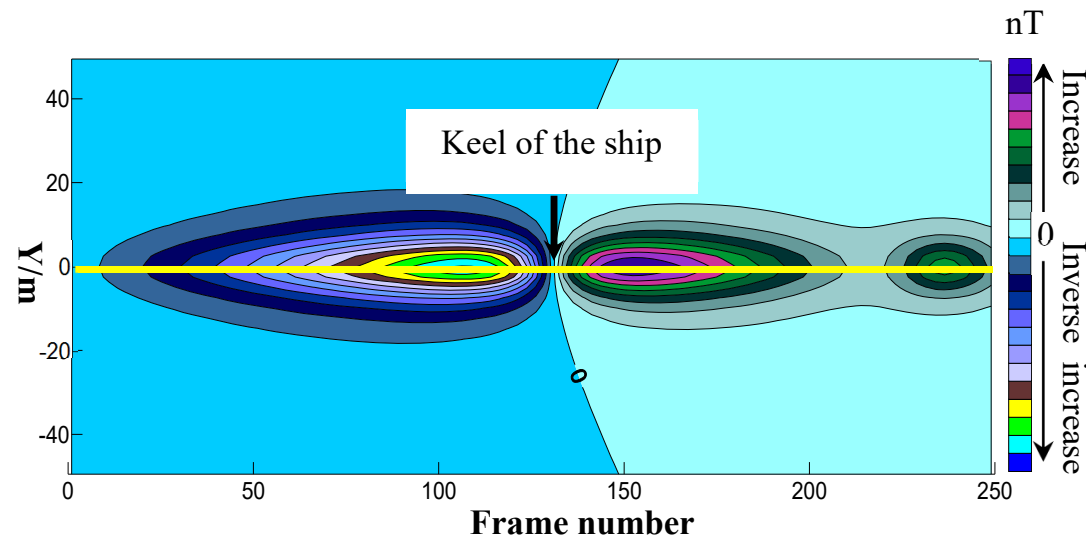

Figure 10: CRM field distribution after anodes position and current optimization.

\section{Conclusions}

An analysis based on BEM can reasonably predict and optimize the potential distribution and minimize the magnetic signature of a vessel. 
Anode position optimization is an effective approach to control CRM signature; for the ship considered, the signature was least when the anodes were symmetrically installed on the $130^{\text {th }}$ frame and the $232^{\text {nd }}$ frame.

The magnetic signature was reduced by about twenty-five percent through the applied anode position and output current optimization.

\section{References}

[1] Santana Diaz, E., Adey, R., Baynham, J. \& Pei, Y.H, Optimisation of ICCP systems to minimise electric signatures. MARELEC Conference, Stockholm, 2001.

[2] Adey, R. \& Baynham, J., Predicting corrosion related electrical and magnetic fields using BEM, UDT Europe 2000: Undersea Defence Technology Conference and Exhibition, London, 2000.

[3] Santana Diaz, E. \& Adey, R., Optimisation of the performance of an ICCP system by changing current supplied and position of the anode. WIT Transactions on Modelling and Simulation, 32, pp. 475-485, 2002.

[4] Santana Diaz, E. \& Tims, R., A complete underwater electric and magnetic signature scenario using computational modelling. Proceedings of the Marine Electromagnetics Conference (MARELEC), Amsterdam, 2006.

[5] Santana Diaz, E. \& Adey, R., Optimising the location of anodes in cathodic protection systems to smooth potential distribution. Advances in Engineering Software, 36(9), pp. 591-598, 2005.

[6] Santana Diaz, E. \& Adey, R., Predicting the coating condition on ships using ICCP system data, Tri-Service Corrosion Conference, Las Vegas, 2003. 